\title{
Comparison of Optical and Gravimetric Methods for Detection of Chymotrypsin
}

\author{
Ivan Piovarci ${ }^{1, *}$, Tibor Hianik ${ }^{1}$ and Ilia N. Ivanov ${ }^{2}$ \\ 1 Department of Nuclear Physics and Biophysics, Faculty of Mathematics, Physics and Informatics, \\ Comenius University, Mlynska Dolina F1, 84248 Bratislava, Slovakia; tibor.hianik@fmph.uniba.sk \\ 2 Center for Nanophase Materials Sciences, Oak Ridge National Laboratory, Oak Ridge, TN 37831, USA; \\ ivanovin@ornl.gov \\ * Correspondence: piovarci.i@gmail.com
}

Received: date; Accepted: date; Published: date

\begin{abstract}
Proteases play an important role in various biological as well as in the dairy industrial processes. In this work we focused on chymotrypsin which is an important protease in human digestion. Chymotrypsin also cleaves milk proteins which makes it useful in the study of activity of milk proteases. In this work we focused on detection of chymotrypsin using optical and gravimetric techniques, their peculiarities, and the limit of detection (LOD) $(0.15 \pm 0.005 \mathrm{nM}$ and $0,64 \pm 0.31 \mathrm{nM}$ for optical and gravimetric methods, respectively). While the optical detection is easier to implement, the gravimetric method is more robust for sample preparation. Both methods could represent a novel assay for detection of chymotrypsin and other milk proteases.
\end{abstract}

Keywords: QCM; chymotrypsin; $\beta$-casein; nanoparticles

\section{Introduction}

Proteases represent a very wide and important group of enzymes for various biological systems. Their proteolytic activity determines final structure and utility of proteins [1]. Because of this, proteases also take part in different pathological processes such as atherosclerosis, malabsorption syndrome, lymphocyte dysfunction, cancer, and serve an important role in human digestion [2,3]. Chymotrypsin, one of the proteases present in human digestion system, plays a significant role in protein cleavage in the intestines. Together with trypsin, in the zymogen form, chymotrypsinogen is ejected into the duodenum, where trypsin cleaves it into the active form [4]. Chymotrypsin is a serine protease that cleaves peptide bonds by hydrolysis at the location of tryptophan, tyrosine, phenylalanine or leucin [5]. Its activity is closely tied to the activity of trypsin, which, along with plasmin, is an important enzyme in human milk. Activity of plasmin ties to the quality of milk. Higher protease activity cleaves the proteins, mainly caseins, in the micelles in milk and affects the milk shelf-life or cheese yield and flavor [6]. Chymotrypsin and trypsin both cleave caseins and therefore biosensors made based on the cleavage of caseins are sensitive for both proteases and plasmin.

In pathology and medicine chymotrypsin has also anti-inflammatory effects and has been successfully used to reduce post-operation complication after cataract surgery [7]. Measuring chymotrypsin activity in medicine can help during differential diagnosis of acinar and endocrinal neoplasms [8].

Thus, development of sensitive, inexpensive, fast, and easy to use methods for detection of chymotrypsin or other milk proteases would be beneficial for both medicine and dairy. The assay that fulfills these requirements is not yet available. The protease detection is currently based on the 
detection of $\alpha$-amino groups cleaved from the protein substrate using optical or high-performance liquid chromatography (HPLC) methods. The method that can be used for fast analysis of the protease concentration is based on Enzyme Linked Immunosorbent Assay (ELISA) with limit of detection (LOD) of about $0.5 \mathrm{nM}$ for chymotrypsin $[9,10]$. However, above mentioned method does not allow to study kinetics of substrate digestion.

In this paper we propose two efficient methods for chymotrypsin detection: optical and gravimetric. Gravimetric method is based on measurement of the resonant frequency of shearing oscillations of AT-cut quartz crystal as well as motional resistance, $\mathrm{R}_{\mathrm{m}}$, and is known as thickness shear mode method (TSM). The protease substrate such as $\beta$-casein or short specific peptides are immobilized at thin gold layers sputtered at TSM transducer. High frequency voltage typically in the range of $5-20 \mathrm{MHz}$ induces shearing oscillations of the crystal. The fundamental resonance frequency of the crystal, $\mathrm{f}_{0}$, depends on the physical properties of the quartz (density, shearing modulus and thickness), viscosity of the medium to which the crystal surface is exposed as well as on the molecular interactions at the surface. The $\mathrm{Rm}_{\mathrm{m}}$ value is sensitive to shearing viscosity that is due to molecular slip between the protein layer and surrounding water environment. Using Sauerbrey Equation (1) [11], one can link the change in resonant frequency to the mass bound to the surface of the electrode.

$$
\Delta \mathrm{f}=-2 \mathrm{f}_{\mathrm{o}}{ }^{2} \Delta \mathrm{m} / \mathrm{A}\left(\mu_{\mathrm{q} Q \mathrm{q}}\right)^{1 / 2},
$$

where $f_{0}$ is the fundamental resonant frequency $(\mathrm{Hz}), A$ is the active crystal area (usually $\left.0.2 \mathrm{~cm}^{2}\right), \rho_{q}$ is quartz density $\left(2.648 \mathrm{~g} . \mathrm{cm}^{-3}\right), \Delta m$ is the mass change $(\mathrm{g}), \rho_{q}$ is the shear modulus of the crystal $\left(2.947 \times 10^{11} \mathrm{~g} \cdot \mathrm{cm}^{-1} \mathrm{~s}^{-2}\right)$. This equation is valid only for rigid layer at vacuum. In liquid environment and for relatively soft layers the viscosity contribution can be estimated by measurements of $\mathrm{Rm}$.

In this work we modified the surface of the crystal with $\beta$-casein a natural substrate for chymotrypsin. The resulting mass added to the sensor caused the change of the frequency $f$ and changes of motional resistance, $\mathrm{R}_{\mathrm{m}}$. Further changes of these values can be monitored following addition of chymotrypsin which leads to cleavage of the $\beta$-casein. The mass-sensitive method has been already used for detection of other proteases. Gronewold [12] monitored activity of bacterial proteases and esperase using proteins from bovine fetal serum. Poturnayova et. al. used $\beta$-casein layers to detect activity of plasmin and trypsin with LOD around 0,65 nM [13]. In another work of Tatarko et. al. the multiharmonic quartz crystal microbalancee (QCM) allowed detection of trypsin and plasmin with LOD of $0.2 \mathrm{nM}$ and $0.5 \mathrm{nM}$, respectively. Machine learning algorithm applied in this work allowed to distinguish these two proteases within 2 min [14].

We also applied optical method based on measurement absorbance of the dispersion of gold nanoparticles (AuNPs) coated by $\beta$-casein and 1-mercaptohexanol (MCH). AuNPs reveal surface plasmon resonance (SPR) effect, which can be measured using UV-Vis spectroscopy. The surface plasmon resonance (SPR) effect arises when the oscillating electromagnetic field of light rays get into contact with the free electrons in metallic nanoparticles and induces their coherent oscillation. The maximum amplitude of this oscillation happens at a specific wavelength called SPR. The SPR position and intensity depends on various factors like metal type, particle size, shape, structure, and dielectric constant of surrounding medium [15]. The absorbance of gold nanoparticles has a maximum in visible spectrum depending on the surrounding medium and on the distance between nanoparticles. AuNPs are rather popular in biosensing applications. In the work by Diouani AuNPs modified with casein were used to detect Leishmania infantum using amperometric methods [16]. Chen et al., the modified AuNPs with trypsin-specific peptide sequence [17]. After the trypsin cleavage the gold nanoparticles aggregated which was visible by measuring UV-Vis spectrum. The detection limit of this method was $5 \mathrm{nM}$. Guaris et al., used dithiols to measure thrombin activity [18]. In this work a presence of substrate for thrombin resulted in aggregation of AuNPs, while addition of thrombin disaggregated the nanoparticles (LOD for thrombin detection was around 5 $\mathrm{nM}$ ). Since AuNPs can be easily modified by thiol-group-containing peptides, this method makes it possible to prepare substrates with well-observable absorption properties. In or work we modified the gold nanoparticles with $\beta$-casein and $\mathrm{MCH}$. The $\beta$-casein protects the AuNPs from aggregation 
induced from the loss due to presence of $\mathrm{MCH}$. Addition of the chymotrypsin and subsequent cleavage of the $\beta$-casein caused nanoparticles aggregation due to lost of protective shell. This effect was possible to observe by measuring UV-Vis spectra of nanoparticle dispersion.

\section{Materials and Methods}

\subsection{Chemicals}

The chemicals needed to prepare the piezocrystal and gold nanoparticles were purchased from Sigma-Aldrich Germany, the chemicals needed to clean the crystals were purchased from Slavus Slovakia. Deionized water was distilled directly at the flask using a Purelab Classic UV instrument (Elga, England). All standard chemicals, such as auric acid (HAuCl4), sodium citrate, $\beta$-casein, 1-mercaptohexanlo (MCH), PBS tablets, 11-mercaptoundecanoic acid (MUA), $\mathrm{N}$-(3-dimetylaminopropyl)-N'-etylcarbodiimid (EDC), N-hydroxisuccinimid (NHS), $\alpha$-chymotrypsin, $\mathrm{NaOH}, \mathrm{HC} \mathrm{NaOH}, \mathrm{NH}_{3}, \mathrm{H}_{2} \mathrm{O}_{2}$ were of p.a. grade.

\subsection{Optical Method}

AuNPs were prepared by modified citrate method [19]. In short, we prepared $100 \mathrm{~mL}$ of 0,01\% chloroauric acid $\left(\mathrm{HAuCl}_{4}\right)$ and brought it to boil. We then added $5 \mathrm{~mL}$ of $1 \%$ sodium tris-citrate and left the solution boiling while stirring until it turned deep red. We then left as prepared AuNPs to cool down and stored them in the dark. To modify the gold nanoparticles with casein, we added 2 $\mathrm{mL}$ of $0.1 \mathrm{mg} / \mathrm{mL} \beta$-casein into $18 \mathrm{~mL}$ of the AuNPs solution. After $2 \mathrm{~h}$ of incubation at room temperature without stirring, the gold nanoparticles were further incubated with $200 \mu \mathrm{L}$ of $1 \mathrm{mM}$ $\mathrm{MCH}$ overnight for approximately $18 \mathrm{~h}$. $\mathrm{MCH}$ is important as it removes the surface charge of nanoparticles and thus protect them from aggregation. Addition of $\mathrm{MCH}$ leads to a color change to violet. However, nanoparticles (NPs) are protected from aggregation also due to the presence of $\beta$-casein layer. Addition of chymotrypsin caused cleavage of $\beta$-casein and as a result the NPs aggregated. This was reflected by changes of the color of solution to blue and then it becomes colorless. For the experiments, we prepared $0.95 \mathrm{~mL}$ of NPs. Chymotrypsin was dissolved in deionized water and $0.05 \mathrm{~mL}$ of chymotrypsin from stock solution was added to each cuvette. The concentration of chymotrypsin in cuvettes was $0.1 ; 0.3 ; 0.5 ; 0.7 ; 1 ; 5$ and 10 at $1 \mathrm{~mL}$ total volume of solution. We also used a reference cuvette where only $0.05 \mathrm{~mL}$ of protease-free water was added to the AuNPs solution. We measured the spectra of the AuNPs before protease addition ( $0 \mathrm{~min})$, just after protease addition (approximately $30 \mathrm{~s}$ ) and then every $15 \mathrm{~min}$ and up to $60 \mathrm{~min}$. The measurement was repeated 3 times. We multiplied the value of absorbance at time $t=0$ by the dilution factor to correct the changes in absorbance intensity caused by the initial protease addition. Absorbance was measured by UV-1700 spectrophotometer (Shimadzu, Japan). Scheme of AuNPs modification and chymotrypsin cleavage is presented in Figure 1. 


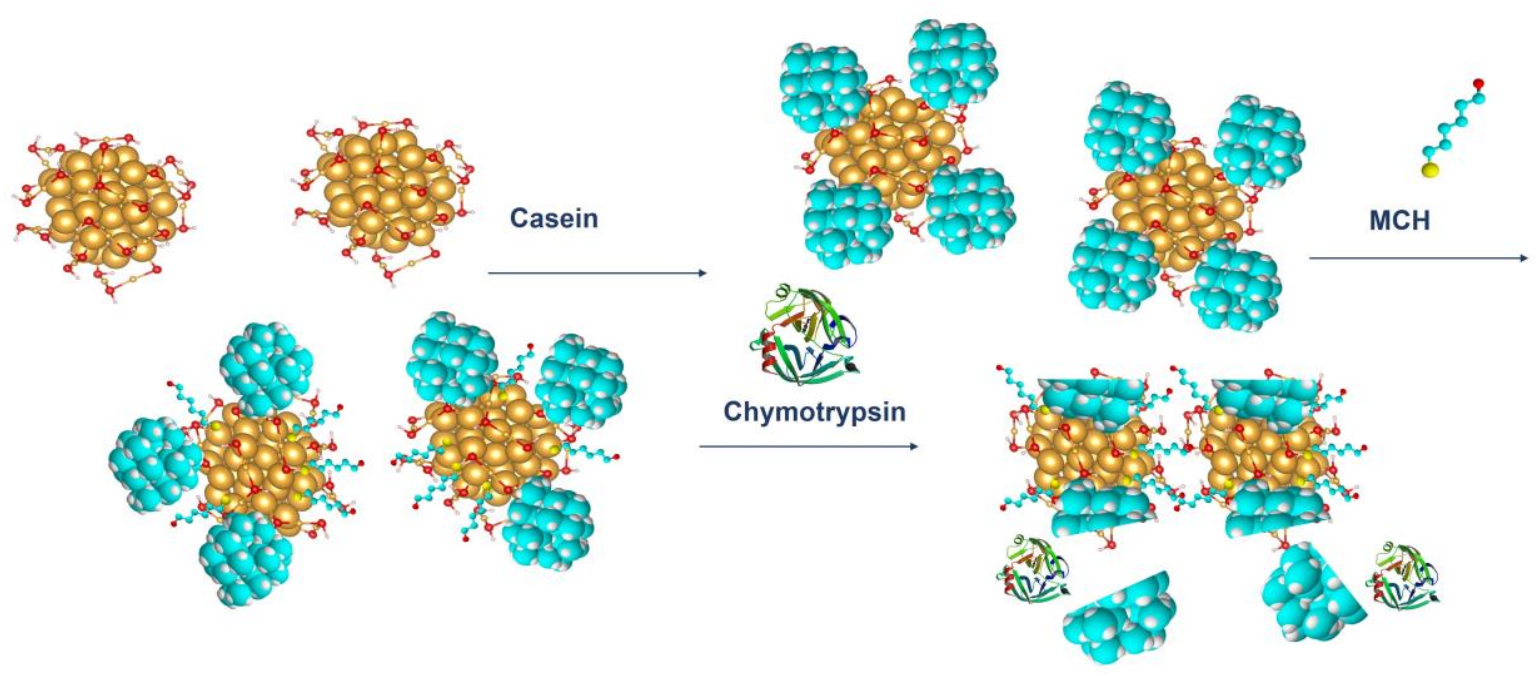

Figure 1. Scheme of AuNPs modification and chymotrypsin cleavage.

\subsection{Thickness Shear Mode (TSM) Method}

The acoustic TSM sensor was prepared on a gold quartz crystal layer for QCM (8 MHz, ICM, Oklahoma, USA, electrode area: $\left.\mathrm{A}=0.2 \mathrm{~cm}^{2}\right)$. First, the crystal was carefully cleaned as follows. It has been exposed to a basic piranha solution $\left(\mathrm{H}_{2} \mathrm{O}_{2}: \mathrm{NH}_{3}: \mathrm{H}_{2} \mathrm{O}=1: 1: 5 \mathrm{~mL}\right)$. The crystals were immersed for $25 \mathrm{~min}$ in this solution, in beakers in a water bath set at approximately $75{ }^{\circ} \mathrm{C}$. Subsequently, the crystals were withdrawn, rinsed with distilled water, and returned to the beaker with a new dose of piranha solution on the reverse side of the crystal. This was repeated three times. On the last extraction, the crystals were washed three times with distilled water and then washed with ethanol and placed in a bottle with ethanol for storage at room temperature. We applied this procedure for each crystal and then used it repeatedly for measurement. The clean crystal has been incubated overnight for $16-18 \mathrm{~h}$ at room temperature with $2 \mathrm{mM}$ MUA dissolved in ethanol. MUA is a carboxylic acid with a sulfide group -SH. The sulfide moiety interacts with the gold on the crystal to form a self-assembled layer. After incubation, the crystal was washed with ethanol, distilled water, and $20 \mathrm{mM}$ EDC and $50 \mathrm{mM}$ NHS were applied for $25 \mathrm{~min}$. These substances react with the carboxyl moiety of MUA and activate them to form a covalent bond with other amino acids. Subsequently, we cleaned the crystal again, dried it with nitrogen and placed it in a cell. The cell was filled with PBS buffer using a Genie plus 2011 step pump (Kent Scientific, USA) at a flow rate of $200 \mu \mathrm{L} / \mathrm{min}$. After filling the cell, we switched the flow to the rate of $50 \mu \mathrm{L} / \mathrm{min} .1 \mathrm{mg} / \mathrm{mL}$ of $\beta$-casein dissolved in PBS was then added to the MUA layer. After $35 \mathrm{~min}$, only pure PBS was added to remove of the unbound fragments. $\beta$-casein layer formation and in some cases EDC/NHS layer activation was recorded using an RQCM instrument (Maxtek, USA).

After binding of $\beta$-casein to the electrode surface and stabilizing the frequency (washing out all unbound residues), we applied chymotrypsin to the crystal at concentrations of $1 \mathrm{pM} ; 10 \mathrm{pM} ; 100$ $\mathrm{pM} ; 1 \mathrm{nM} ; 10 \mathrm{nM}$ and $20 \mathrm{nM}$. After 35 min of chymotrypsin application, the PBS let into the cell until the resonant frequency stabilized. The change in resonant frequency from chymotrypsin application to stabilization in PBS was taken as the amount of casein cleaved from the layer. After lower concentrations (1 pM, $10 \mathrm{pM}, 100 \mathrm{pM})$, we applied a higher concentration of chymotrypsin (at least at a concentration 2 orders of magnitude higher). In such measurements, we analyzed the degree of cleavage as the change in frequency from the initial state to a steady value. All measurements were performed at PBS, $7.4 \mathrm{pH}$.

For both optical and gravimetric methods, the LOD was determined using equation: LOD = $3,3^{*}(\mathrm{SD}) / \mathrm{S}$, where SD is standard deviation of the sample with lowest concentration and $\mathrm{S}$ is slope calculated from fit of linear part o calibration curve. Scheme of modification of TSM piezo and chymotrypsin cleavage is presented in Figure 2. 


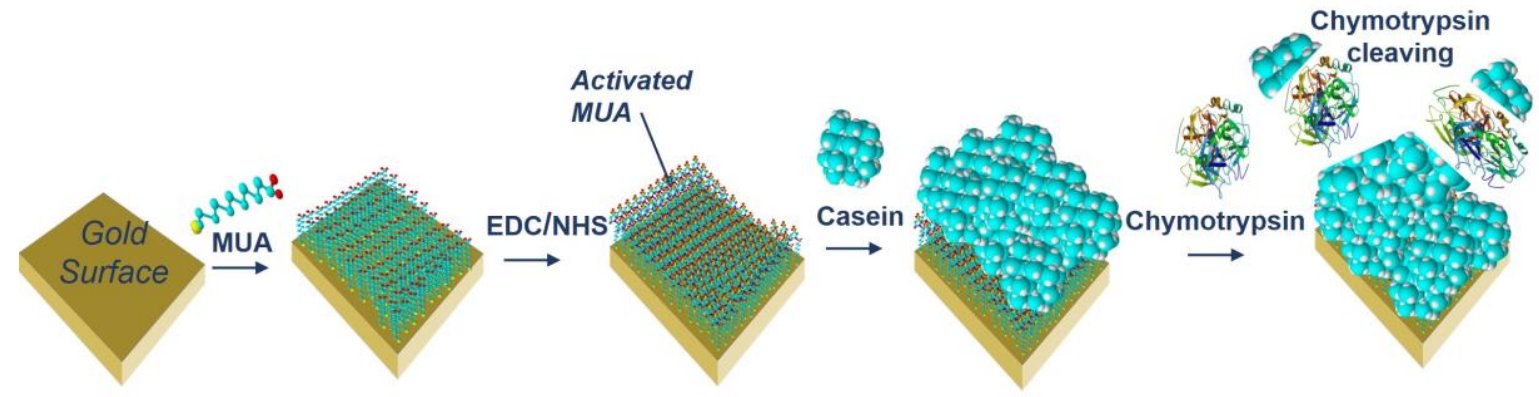

Figure 2. Scheme of modification of the piezocrystal and chymotrypsin cleavage.

\section{Results and Discussion}

\subsection{Detection of Chymotrypsin by Optical Method}

We modified the gold nanoparticles with $\beta$-casein and subsequently with $\mathrm{MCH}$. In Figure 3 we can see change in the absorption spectra after each step. After modification with $\beta$-casein we can see shift of the maximum of absorbance by around $5 \mathrm{~nm}$ and a slight increase in absorbance. After addition of $\mathrm{MCH}$ the peak becomes broader and a significant shift of around $60 \mathrm{~nm}$ occurred. This suggests that the nanoparticles getting closer together and are starting to aggregate. This is due to the $\mathrm{MCH}$ replacing the $\beta$-casein protective layer and in turn making the nanoparticles closer to each other.

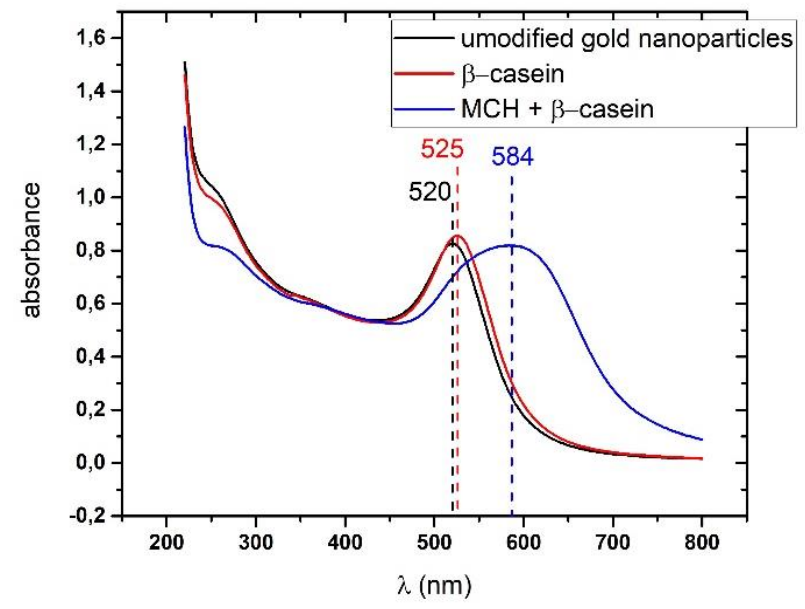

Figure 3. Absorption spectra for gold nanoparticles (AuNP) prepared by Turkevich method: unmodified (black), modified by $\beta$-casein (red) and further modified by $\mathrm{MCH}$ (blue).

We measured the changes of absorbance spectra of AuNPs suspension during the chymotrypsin cleavage at $0 \mathrm{~min} ; 0.5 \mathrm{~min} ; 15 \mathrm{~min} ; 30 \mathrm{~min}$ and $60 \mathrm{~min}$. Changes in spectra over time for two different concentration of chymotrypsin are presented in Figure 4 . At relatively low concentration of chymotrypsin $(0.1 \mathrm{nM})$ we did not observe significant changes of the absorbance (Figure 2a). However, at higher chymotrypsin concentration, around $10 \mathrm{nM}$, a substantial red shift of the spectra was observed (up to $615 \mathrm{~nm}$ ). It can be also seen that the absorbance decreased with time. that after the maximum shifting enough the absorbance starts to decrease. 


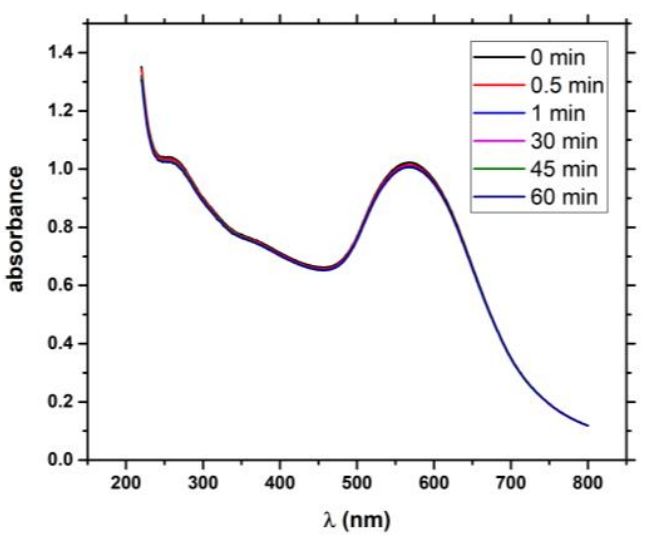

(a)

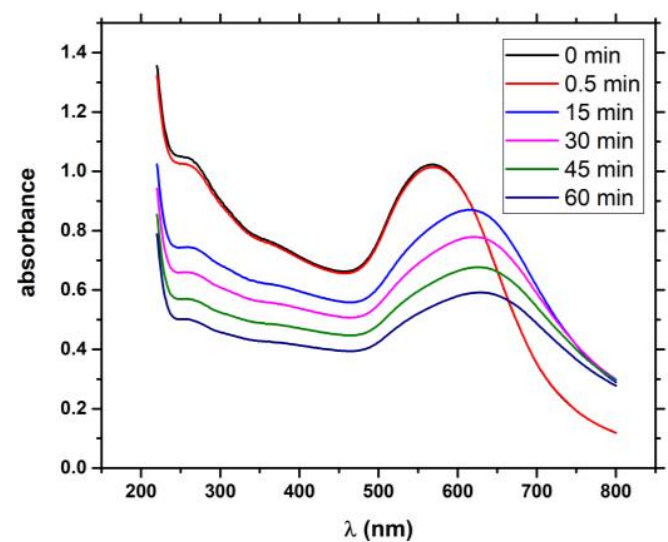

(b)

Figure 4. Changes of absorbance spectra of the suspension of AuNPs modified by $\beta$-casein and MCH in time for (a) 0,1 nM chymotrypsin and (b) $10 \mathrm{nM}$ chymotrypsin.

In Figure 5. we show the change of maximum position and absorbance in time for all concentrations.

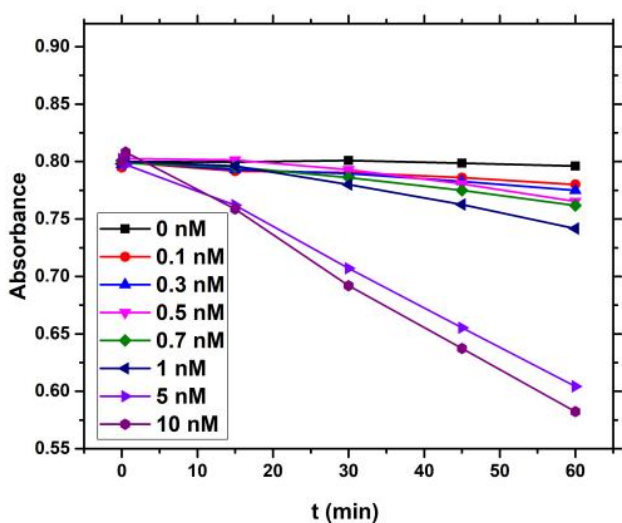

(a)

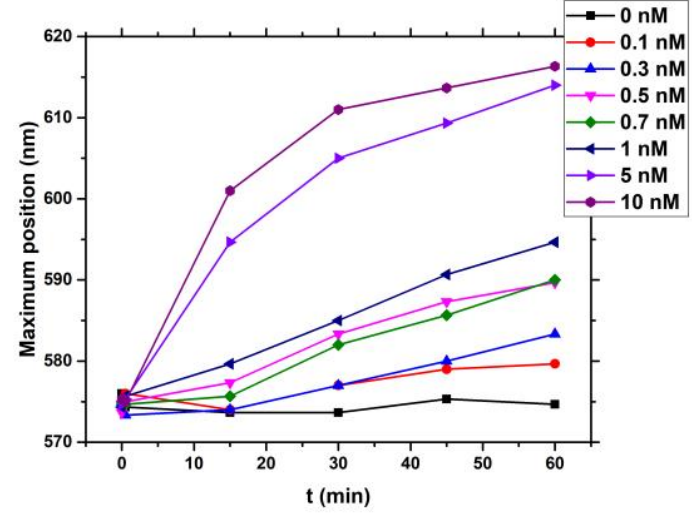

(b)

Figure 5. (a) Changes in the value of absorbance peak vs. time for different chymotrypsin concentrations; (b) changes in maximum position of absorbance peak vs. time for different chymotrypsin concentration in a suspension of AuNPs modified by $\beta$-casein and $\mathrm{MCH}$.

For higher concentration of chymotrypsin, the rate of absorbance increases. The maximum position of absorbance peak shift with time and with increasing chymotrypsin concentration. However, for 5 and $10 \mathrm{nM}$ chymotrypsin the maximum position stabilizes around $615 \mathrm{~nm}$. In order to prepare calibration curve, we fit the change of absorbance with linear curve and then we differentiate this model numerically to obtain the values of $\mathrm{dA} / \mathrm{dt}$. We then use the $\mathrm{dA} / \mathrm{dt}$ value at $\mathrm{t}=$ $0 \mathrm{~min}$ in order to obtain initial rate of the reaction. The calibration curve is presented in Figure 6. 


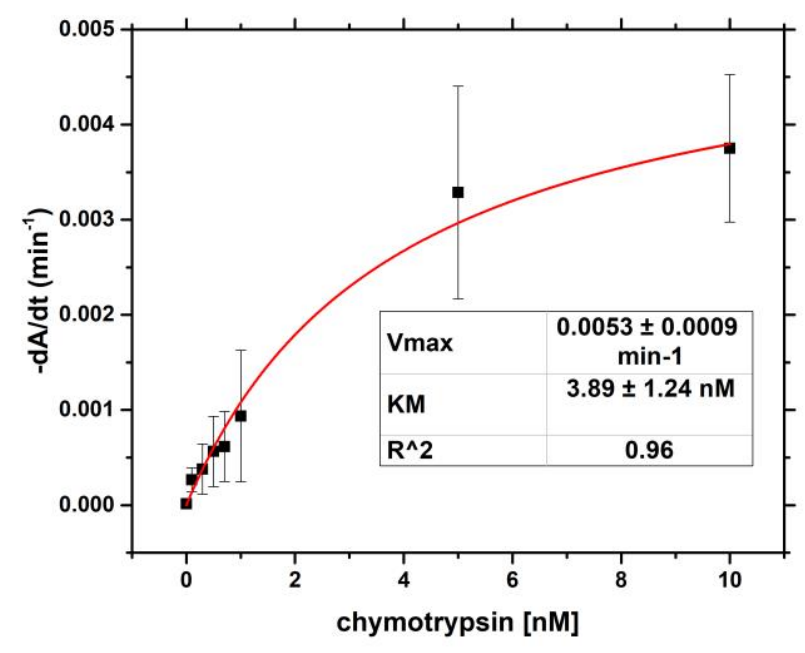

Figure 6. Calibration curve for chymotrypsin with reverse Michaelis-Menten model for optical method. $\mathrm{dA} / \mathrm{dt}$ is numerical derivation of linear model of absorbance change at time $t=0$ and corresponds to the rate of enzyme reaction.

We were able to use reverse Michaelis-Menten model to analyze the obtained data. However instead of substrate concentration, the concentration of chymotrypsin, $c$, has been used in this model: $\mathrm{v}=\mathrm{V} \max \left[\mathrm{c} /\left(\mathrm{K}_{\mathrm{M}}+\mathrm{c}\right)\right]$, where $\mathrm{v}$ and $\mathrm{V} \max$ are the rate and maximum rate of enzyme reaction, respectively, and $\mathrm{K}_{\mathrm{M}}$ is reverse Michaelis-Menten constant. In our case $\mathrm{v}=\mathrm{dA} / \mathrm{dt}$. However, this model is used only formally because different restraints. The main assumptions of excess enzyme and limited substrate concentration is reversed in this case and we instead change the concentration of enzyme while substrate is present in excess, still this method can be used with good approximation [20]. To calculate LOD we used only part of the calibration curve from 0 to $5 \mathrm{nM}$, where the plot of $\mathrm{dA} / \mathrm{dt}$ vs $\mathrm{c}$ is almost linear. The results are shown in Figure 7.

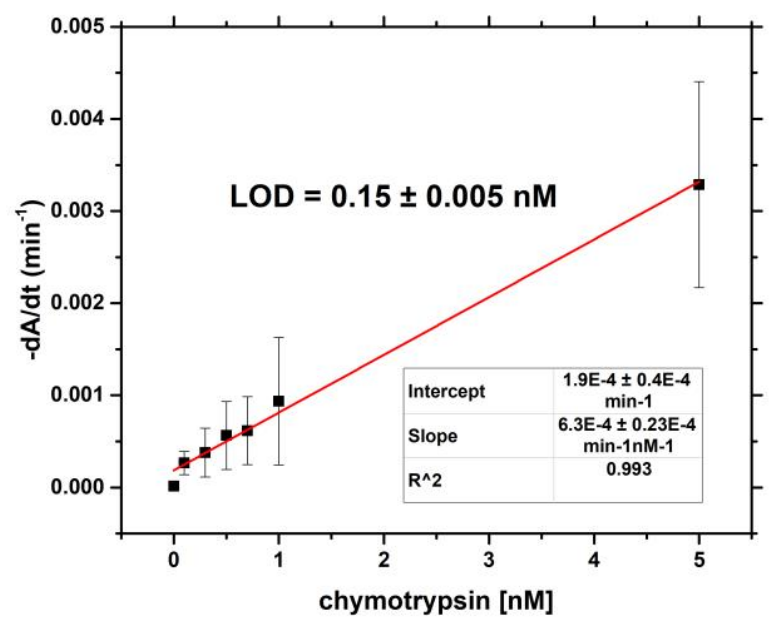

Figure 7. Linear part of calibration curve $-\mathrm{dA} / \mathrm{dt}$ vs. concentration of chymotrypsin for calculation of LOD.

The LOD for optical method of chymotrypsin detection was $0.15 \pm 0.005 \mathrm{nM}$. This value is comparable to that of ELISA method which is around $0.5 \mathrm{nM}$ [10]. However, in contrast with ELISA that requires specific antibodies, the method based on AuNPs is much easier and faster. Detection of chymotrypsin takes only $30 \mathrm{~min}$. In addition, once the AuNPs are prepared and modified by 
$\beta$-casein and $\mathrm{MCH}$, only one step is necessary for detection of chymotrypsin concentration in unknown sample. The disadvantage of this method is that only transparent samples can be used for detection. This restriction can be avoided using surface sensitive gravimetric method.

\subsection{Detection of Chymotrypsin ba Gravimetric Method}

For the gravimetric method we first modified the surface of the piezocrystal with adsorbed MUA by $\beta$-casein. By monitoring resonant frequency and motional resistance it is possible to study all steps of the preparation of sensing surface. This is presented on Figure 8.

After the activation of carboxylic groups of MUA with EDC/NHS the frequency shifted only slightly. After the $\beta$-casein addition we can see a fast drop of resonant frequency of about $170 \mathrm{~Hz}$. After changing the flow with buffer, it can be seen the frequency increase due to the removal of non-specifically bound $\beta$-casein. The resulting frequency shift after washing the surface corresponds to $120 \mathrm{~Hz}$. From Sauerbrey equation we can calculate the change in mass on biosensor, which corresponds to about $165 \mathrm{ng}$ of mass added. With the knowledge of the molecular weight of $\beta$-casein $M_{w}=24 \mathrm{kDa}$ we can calculate number of moles on the sensor and surface density $\Gamma=34,5 \mathrm{pM} / \mathrm{cm}^{2}$. From changes in motional resistance $\mathrm{R}$ we can assess the change in viscous properties of the surface. Since the resistance decreases and increases proportionally to the change of frequency, the change in motional resistance is caused mainly just by added weight so we can assume that the casein layer is not rigit.

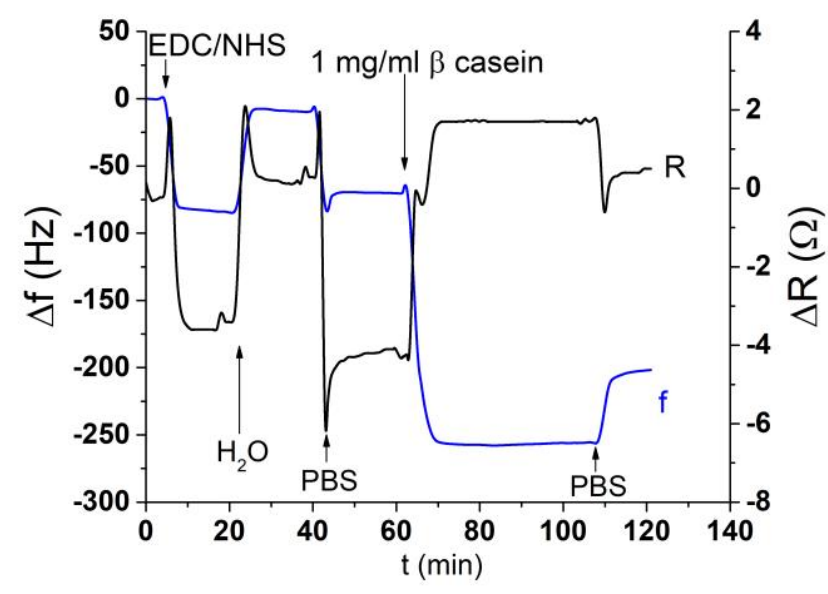

Figure 8. Kinetics of resonant frequency, $f$ (blue) and dynamic resistance, $R_{m}$ (black) changes during modification of piezocrystal by $\beta$-casein. The carboxylic groups of MUA that was chemisorbed at the crystal were first activated by EDC/NHS. The moments of addition of various compounds as well as washing the surface by water and PBS are shown by arrows.

After $\beta$-casein is bound to the surface, we can study its cleavage by different concentrations of chymotrypsin under flow condition for $35 \mathrm{~min}$. Example of the changes of resonant frequency and dynamic resistance following addition of $10 \mathrm{nM}$ chymotrypsin are shown in Figure 9. 


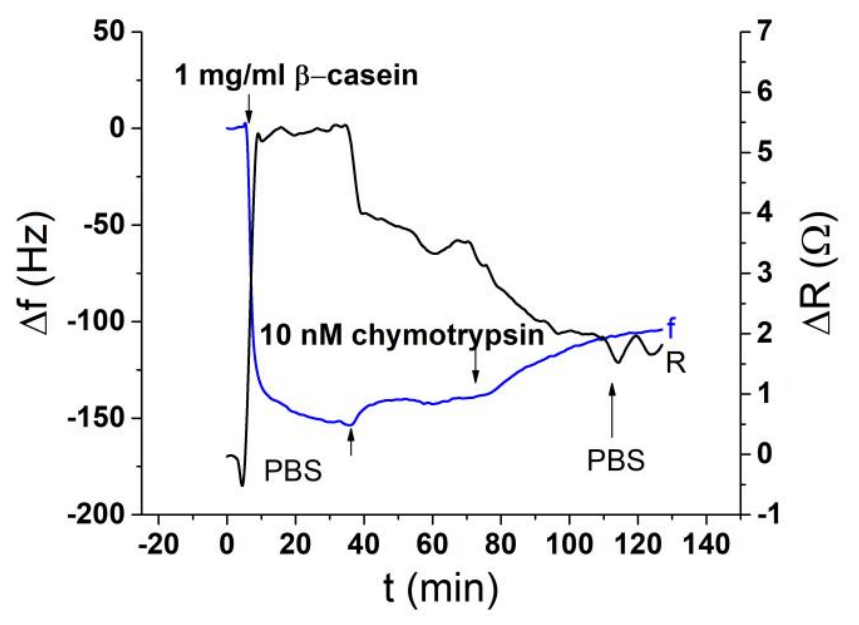

Figure 9. Kinetics of resonant frequency, $\mathrm{f}$ (blue) and dynamic resistance, $\mathrm{R}$ (black) changes following modification of piezocrystal by $\beta$-casein and addition of $10 \mathrm{nM}$ of chymotrypsin.

Based on the changes of the frequency we constructed calibration curve for different concentration of chymotrypsin (Figure 10a) and for determination of LOD we also presented calibration curve at low concentration range where the dependence is almost linear (Figure 10b). The LOD for gravimetric method was $0.64 \pm 0.31 \mathrm{nM}$. This value is higher than that reported in ELISA methods and optical method used here. It is also more difficult to work with flow during the measurement and pressure changes caused by handling the flow cell can affect the results. This method is, however, robust to different components in sample such as added fat present naturally in milk. Timewise this method takes similar time to spectrophotometric method.
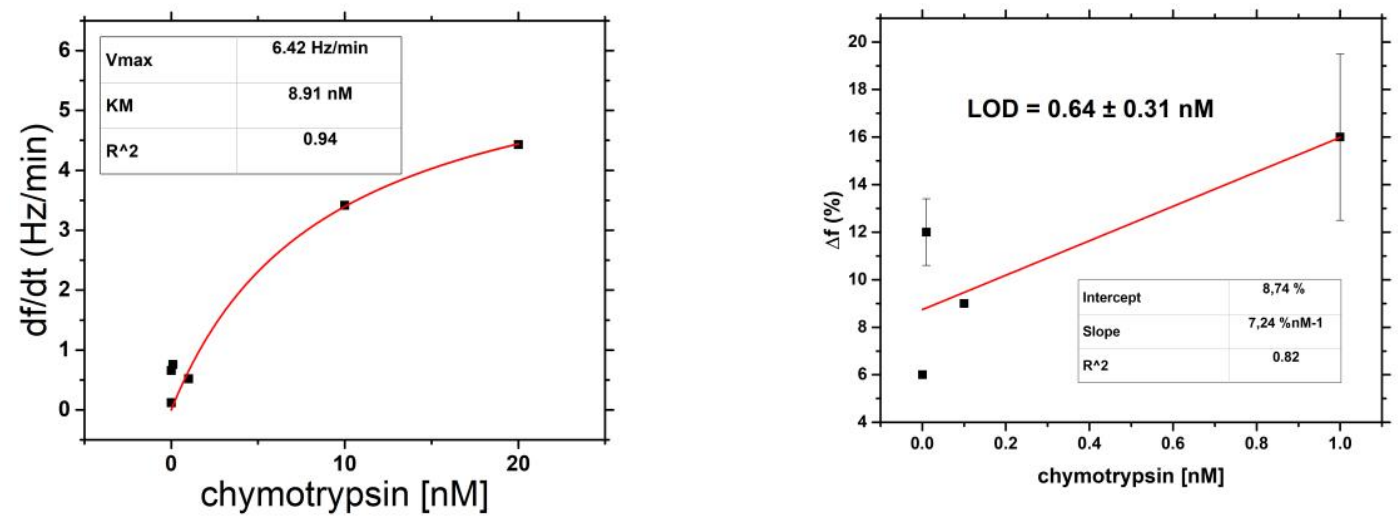

Figure 10. (a) Calibration curve for chymotrypsin fitted by reverse Michaelis-Menten model, $v=$ $\mathrm{df} / \mathrm{dt}$ is first derivation of frequency obtained from kinetic curve. (b) calibration curve: changes in frequency $\left(\Delta \mathrm{f}=\left(\Delta \mathrm{f}_{\text {chymo }}\right) /\left(\Delta \mathrm{f}_{\text {casein }}\right)^{*} 100\right.$ where $\Delta \mathrm{f}_{\text {chymo }}$ is change in frequency after chymotrypsin cleavage and $f_{\text {casein }}$ is frequency change after formation of $\beta$-casein layer) vs. lower chymotrypsin concentration range for determination of LOD.

It is also interesting to compare the $\mathrm{K}_{\mathrm{M}}$ values determined in optical and gravimetric experiments. From the results presented above it can be seen that $\mathrm{K}_{\mathrm{M}}$ value in the case of AuNPs based optical assay $(3.89 \mathrm{nM})$ is about twice lower in comparison with those based on gravimetric measurements $(8.91 \mathrm{nM})$. This can be evidence on better access of $\beta$-casein substrate for chymotrypsin at AuNPs in comparison with those immobilized at the surface of TSM transducer. This effect can probably explain the lower LOD for optical method of chymotrypsin detection with those based on TSM. 


\section{Conclusions}

We determined LOD for both gravimetric (LOD $=0.64 \pm 0.31 \mathrm{nM}$ ) and optical (LOD $=0.15 \pm$ $0.005 \mathrm{nM})$ methods. Optical method showed the best value of LOD even when compared to commercial ELISA (LOD $=0.5 \mathrm{nM}$ ). In terms of time both methods are comparable and take around $30 \mathrm{~min}$ for chymotrypsin determination if the sensors are prepared beforehand. Both methods require sensing assay preparation overnight, however in both cases the AuNPs or gravimetric sensors can be stored long time (more than one month) at $4{ }^{\circ} \mathrm{C}$. In terms of difficulty in operation, the optical methods offer the easier way to measure chymotrypsin. With prepared AuNPs modified by $\beta$-casein and $\mathrm{MCH}$ the optical method requires only one step of protease detection based on measurement of absorbance changes after $30 \mathrm{~min}$, which is easier in comparison with ELISA. In terms of sample usage, the optical method needs only $50 \mu \mathrm{L}$ of sample while the gravimetric method used around $2 \mathrm{~mL}$. One of the advantages of gravimetric method is that it is more robust to "impurities" in sample. If the gold crystal is correctly modified the gravimetric method can be used with natural, not transparent samples containing fat, minerals or other proteins just like in milk. In contrast, optical assay requires transparent sample. Both methods have a distinct advantage and disadvantage over ELISA concerning their specificity. In contrast with ELISA, the optical and gravimetric assay are not specific to the protease. In some cases, this problem can be solved by using specific peptide substrate [13]. This problem could also be overcome using machine learning as evident in the work of Tatarko et. al. [14]. In conclusion both optical and gravimetric methods have advantages and disadvantages in detecting chymotrypsin or other proteases and could represent novel approach for practical application in food industry and medicine for real time monitoring of the protease activity.

Author Contributions: conceptualization, T.H. and I.N.I.; methodology, I.P.; formal analysis, I.P.; investigation, I.P.; resources, T.H. and I.N.I.; writing-original draft preparation, I.P.; writing-review and editing, T.H. and I.N.I.; visualization, I.P.; supervision, T.H. and I.N.I.; project administration, T.H.; funding acquisition, T.H. and I.N.I. All authors have read and agreed to the published version of the manuscript.

Funding: A portion of this research was conducted at the Center for Nanophase Materials Sciences, which is a DOE Office of Science User Facility, project No. CNMS2018-293. This work was funded under European Union's Horizon 2020 research and innovation program through the Marie Skłodowska-Curie grant agreement No 690898 and by Science Agency VEGA, project No. 1/0419/20.

Conflicts of Interest: The authors declare no conflict of interest. The funders had no role in the design of the study; in the collection, analyses, or interpretation of data; in the writing of the manuscript, or in the decision to publish the results.

\section{References}

1. Bond, J.S. Proteases: History, discovery, and roles in health and disease. J. Biol. Chem. 2019, 294, $1643-1651$.

2. Lojda, Z. The importance of protease histochemistry in pathology. Histochem. J. 1985, 17, 1063-1089.

3. Antalis, T.M.; Shea-Donohue, T.; Vogel, S.N.; Sears, C.; Fasano, A. Mechanisms of disease: Protease functions in intestinal mucosal pathobiology. Nat. Clin. Pract. Gastroenterol. Hepatol. 2007, 4, 393-402.

4. Appel, W. Chymotrypsin: Molecular and catalytic properties. Clin. Biochem. 1986, 19, 317-322.

5. Tomlinson, G.; Shaw, M.C.; Viswanatha, T. Chymotrypsin(s). Methods Enzymol. 1974, 34, 415-420.

6. Ismail, B.; Nielsen, S.S. Invited rieview: Plasmin protease in milk: Current knowledge and relevance to dairy industry. J. Dairy Sci. 2010, 93, 4999-5009.

7. Harris, G.S. Alpha-Chymotrypsin in cataract surgery. Can. Med. Assoc. J. 1961, 85, 186-188.

8. Skacel, M.; Ormsby, A.H.; Petras, R.E.; McMahon, J.T.; Henricks, W.H. Immunohistochemistry in the differential diagnosis of acinar and endocrine pancreatic neoplasms. Appl. Immunohistochem. Mol. Morphol. 2000, 8, 203-209.

9. Chen, L.; Daniel, R.M.; Coolbear, T. Detection and impact of protease and lipase activities in milk and milk powders. Int. Dairy J. 2003, 13, 255-275.

10. Chymotrypsin KLISA Available online: http://img2.creative-diagnostics.com/pdf/DEIA10041,Chymotrypsin.pdf (accessed on 7 October 2020). 
11. Kankare, J. Sauerbrey equation of quartz crystal nicrobalance in liquid medium. Langmuir 2002, 18, 7092-7094.

12. Gronewold, T.M. Surface acoustic wave sensors in the bioanalytical field: Recent trends and challenges. Anal. Chim. Acta. 2007, 603, 119-128.

13. Poturnayova, A.; Castillo, G.; Subjakova, V.; Tatarko, M.; Snejdarkova, M.; Hianik, T. Optimization of the cytochrome c detection by acoustic and electrochemical methods based on aptamer sensors. Sens. Act. B: Chem. 2017, 228, 817-827.

14. Tatarko, M.; Muckley, E.; Subjakova, V.; Goswami, M.; Sumpter, B.; Hianik, T.; Ivanov, I. Machine learning enabled acoustic detection of sub-nanomolar concentration of trypsin and plasmin in solution. Sens. Act. B: Chemical. 2018, 272, 282-288.

15. Huang, X.; El-Sayed, M.A. Gold nanoparticles: Optical properties and implementations in cancer diagnosis and photothermal therapy. J. Adv. Res. 2010, 1, 13-28.

16. Diouani, M.F.; Ouerghi, O.; Belgacem, K.; Sayhi, M.; Laouini, D. Casein-conjugated gold nanoparticles for amperometric detection of leishmania infantum. Biosensors 2019, 9, 68.

17. Chen, G.; Yusheng, X.; Zhang, H.; Wang, P.; Cheung, H.; Yang, M.; Sun, H. A general colorimetric method for detecting protease activity based on peptide-induced gold nanoparticle aggregation. RSC Adv. 2014, 4, $6560-6563$.

18. Guarise, C.; Pasquato, L.; De Filippis, V.; Scrimin, P. Gold nanoparticles-based protease assay. Proc. Natl. Acad. Sci. USA 2006, 103, 3978-3982.

19. Kimling, J.; Maier, M.; Okenve, B.; Kotaidis, V.; Ballot, H.; Plech, A. Turkevich method for gold nanoparticle synthesis revisited. J. Phys. Chem. B 2006, 110, 15700-15707.

20. Románszki, L.; Tatarko, M.; Jiao, M.; Keresztes, S.; Hianik, T.; Thompson, M. Casein probe-based fast plasmin determination in the picomolar range by an ultra-high frequency acoustic wave biosensor. Sens. Act. B: Chem. 2018, 275, 206-214.

Publisher's Note: MDPI stays neutral with regard to jurisdictional claims in published maps and institutional affiliations.

(C) 2020 by the authors. Submitted for possible open access publication under the terms and conditions of the Creative Commons Attribution (CC BY) license (http://creativecommons.org/licenses/by/4.0/). 